\title{
Original Research \\ COUP-TFI deletion affects angiogenesis and apoptosis related gene expression in mouse placenta: results of an explorative study
}

\author{
Luigi Viola ${ }^{1}$, Stefania Marzinotto ${ }^{2}$, Michele Bertacchi ${ }^{3}$, Ambrogio P Londero ${ }^{4,5, *}$, \\ Maria Orsaria $^{6}$, Serena Bertozzi ${ }^{5,7}$, Lorenza Driul ${ }^{4}$, Carla Di Loreto ${ }^{8}$, Michèle Studer ${ }^{3}$, \\ Laura Mariuzzi ${ }^{8}$, Arrigo Fruscalzo ${ }^{9, *}$ \\ ${ }^{1}$ Department of Precision Medicine, University of Campania “L. Vanvitelli”, 80138 Naples, Italy \\ ${ }^{2}$ Department of Laboratory Medicine, ASUFC, 33100 Udine, Italy \\ ${ }^{3}$ Université Côte d'Azur (UCA), CNRS, Inserm, iBV, 06108 Nice, France \\ ${ }^{4}$ Obstetrics and Gynecology, AOU S.M. della Misericordia, University of Udine, 33100 Udine, Italy \\ ${ }^{5}$ Ennergi Research (non-profit organization), via Talmassons1, 33050 Lestizza (UD), Italy \\ ${ }^{6}$ Institute of Surgical Pathology, Department of Laboratory Medicine, ASUFC, 33100 Udine, Italy \\ ${ }^{7}$ Clinic of Surgery, AOU Santa Maria della Misericordia, University of Udine, 33100 Udine, Italy \\ ${ }^{8}$ Institute of Surgical Pathology, Department of Medicine - DAME, University of Udine, 33100 Udine, Italy \\ ${ }^{9}$ Clinic of Obstetrics and Gynecology, University Hospital of Fribourg, 1752 Villars sur Glane, Fribourg, Switzerland \\ *Correspondence: arrigo.fruscalzo@h-fr.ch (Arrigo Fruscalzo); ambrogio.londero@gmail.com (Ambrogio P Londero) \\ Academic Editor: Mahmoud S. Ahmed \\ Submitted: 26 July 2021 Revised: 26 August 2021 Accepted: 13 September 2021 Published: 10 January 2022
}

\begin{abstract}
Background: Chicken Ovalbumin Upstream Promoter-Transcription Factor I (COUP-TFI) is a member of the steroid/thyroid nuclear receptor superfamily. The aim of this study was to investigate whether absence of this gene affects placental development and fetal growth in a COUP-TFI knockout mouse model. Methods: Placentas of COUP-TFI-knockout (COUP-TFI KO) and wild-type (WT) were collected at 18.5 days post-coitum. The expression level of the following genes known to be involved in different key molecular pathways was evaluated: BCL2 Associated X (Bax) and B-cell lymphoma 2 (Bcl-2) (apoptosis), p21, p53 and $\alpha$ subunit of inhibin (INHA) (proliferation and apoptosis), vascular endothelial growth factor A $(V E G F-A)$, placental growth factor $(P l G F)$, hypoxia-inducible factor 1-alpha $(H I F 1 \alpha)$, Fms related receptor tyrosine kinase 1 (Flt-1), and endoglin (ENG) (angiogenesis). Mouse litter weight at birth was also assessed. Results: RT-qPCR analysis showed increased mRNA expression of VEGF-A and Bax in placental tissue of COUP-TFI $K O$ mice compared to $W T$ mice. We also found a loss in the positive correlation between $B c l-2$ and $I N H A$, p21 and ENG, as well as HIFl $\alpha$ and Flt-1 mRNA expression in COUP-TFI mutants. Finally, KO mice were lighter than WT littermates (respectively, the mean weight of COUP-TFI KO mice was 1.3 grams, \pm 0.13 , compared to $1.6 \mathrm{~g}, \pm 0.14$ of WT mice, $p<0.05)$. Conclusions: Our results show that $C O U P-T F I$ deletion is associated with a lower birth weight in mice and increased placental transcript expression of pro-apoptotic $B a x$ and pro-angiogenetic $V E G F-A$ genes.
\end{abstract}

Keywords: COUP Transcription Factor I; Nr2f1/NR2F1; Mouse; Placenta; Angiogenesis; Apoptosis

\section{Introduction}

At the basis of the development of a viable pregnancy in mammals there is the adhesion and implantation of a blastocyst to the endometrial epithelium. While the inner cell mass of the blastocyst will form the embryo, the outer layer, called the trophoblast, further develops into the maternal decidua and gives rise to the placenta. This latter represents the pivotal organ linking the developing embryo to the mother, providing the necessary oxygen supply and nutrient exchange [1]. Proliferation, apoptosis, and angiogenesis are crucial mechanisms involved in the correct development and remodeling of all the complex structures that make up the placenta. Impairments of this processes can result in several complications, including a deficient growth of ongoing pregnancy [2].
Chicken
Ovalbumin

Transcription Factor I (COUP-TFI), also known as nuclear receptor subfamily 2 , group $\mathrm{F}$, member $1(N R 2 F 1)$ is an orphan nuclear receptor factor and member of the steroid/thyroid hormone receptor superfamily, mainly expressed in the central and peripheral nervous systems. The mammalian COUP-TFI plays a key role during metabolic homeostasis as well as organogenesis through cell fate determination, differentiation, proliferation, and apoptosis [3,4]. Overall, the high conservation of amino acid sequence between species suggests vital evolutionary conserved functions worth being explored, also when considering the process of placental development [5-7].

Earlier studies conducted on human placenta highlight the potential key role played by COUP-TFI. An insilico investigation of transcriptional profile obtained using a microarray approach revealed that COUP-TFI was 
highly associated with self-renewal and differentiation of human chorionic trophoblast progenitor cells [8]. Furthermore, COUP-TFI was indicated in a meta-analysis among the genes that seems to play a role in the development of pre-eclampsia, a complication of placental function often related to fetal growth restriction [9]. However, whether and how COUP-TFI could influence the process of placental development has not been evaluated yet.

Compared to the monolayer in human placenta (hemomonochorial), placenta in mice is composed by three trophoblast layers (hemotrichorial) [10]. At embryological (E) day 10.5, when mid-gestation begins, all the layers of the placenta are formed, including the outermost maternal part, called the decidua, and the fetal part with the triple trophoblastic layer [11,12]. Embryonic development ends with the mid-gestation phase at E13.5, thereafter the fetus matures until the time of birth, around day E19.5 [13].

Transgenic COUP-TFI KO mice have been developed to shed more light into COUP-TFI functions [14]. Upon COUP-TFI loss, mice litter show a high incidence of perinatal mortality due to several neuronal malformations, particularly in the glossopharyngeal ganglion, defects in axonal arborization, and loss of cortical patterning due to the absence of thalamocortical connections $[7,15]$.

The aim of this study was to explore whether and how COUP-TFI deletion in mice could interfere with placental development in terms of expression of some genes related to proliferation, apoptosis, and angiogenesis and in terms of neonatal weight at birth.

\section{Materials and methods}

\subsection{Animals}

COUP-TFI KO (COUP-TFI -/-) mice were generated and subsequently genotyped using the following primers, as previously described: forward 5'-CTGCTGTAGGAATCCTGTCTC-3', reverse 5' AATCCTCCTCGGTGAGAGTGG- $3^{\prime}$ and reverse 5'-ACATACACAGCCTGGCCTTGC-3' [14,16,17]. Heterozygous mice (COUP-TFI+/-) were bred together to generate COUP-TFI KO mice. Placentas were collected at 18.5 days post-coitum (dpc). The weight of pups was recorded at birth (post-natal day 0). Embryonic day (E) 0.5 was defined as the midday of the day of the vaginal plug. The study and all mouse experiments were conducted in accordance with the ARRIVE (Animal Research: Reporting of In Vivo Experiments) guidelines and was approved by the local ethical committee in France (CIEPAL NCE/2019-548) [18]. One pregnant female at a time was euthanized by cervical dislocation and placentas were collected from foetus. Ten placentas were analyzed in this study; five of them were wild type and 5 were mutant (COUP-TFI KO). The placentas were separated from the surrounding tissue according to the previously described technique [19]. Subsequently, sagittal central sections of the placentas were used for the analysis [19].

\subsection{Real-time PCR}

All samples were kept on ice during dissection, then quickly transferred into $500 \mu \mathrm{L}$ of $\mathrm{TRIzol}^{\mathrm{TM}}$ Reagent (Thermo Fisher Scientific, Waltham, Massachusetts, USA catalog number: 15596026) and processed for total $R N A$ isolation according to the manufacturers' protocol. $1 \mu \mathrm{g}$ of $R N A$ was reverse transcribed using the SuperScript ${ }^{\circledR}$ III REV transcript Kit (Thermo Fisher Scientific, Waltham, Massachusetts, USA catalog number: 12574026). Quantitative $R T-P C R$ reactions were performed by using Roche LightCycler ${ }^{\circledR} 480$ and SSOADV UNIVER SYBR GRN SMX 500 (BIO-RAD, Hercules, California, USA catalog number: 172-5270), according to the manufacturers' protocols.

The following genes have been included as markers for placental development: hypoxia-inducible factor 1alpha $(H i f 1 \alpha)$, endoglin $(E N G)$, Fms related receptor tyrosine kinase 1 (Flt1), placental growth factor $(P l G F)$ and the isoform A of the vascular endothelial growth factor ( $V E G F$ $A$ ), being among the main players involved in angiogenesis pathway, $p 21$ and $p 53$ for cell proliferation and apoptosis, and BCL2 associated X (Bax), BCL2 apoptosis regulator (Bcl2), and $\alpha$ subunit of inhibin (INHA), involved in apoptosis and cell survival. The primers used are displayed in Table 1.

Table 1. List of used primer sequences.

\begin{tabular}{lc}
\hline Primer & Name \\
\hline Sense 5'- CCGAGAATGGGAAGCTTGTC -3' & Gapdh \\
Antisense 5'-TCTCGTGGTTCACACCCATC -3' & Gapdh \\
Sense 5'- CCTTTTTGCTACAGGGTTTCATC -3' & BAX \\
Antisense 5'-AGCTCCATATTGCTGTCCAGTT -3' & BAX \\
Sense 5'- AAGCTGTCACAGAGGGGCTA -3' & Bcl-2 \\
Antisense 5'-TCAGGCTGGAAGGAGAAGATG -3' & $B c l-2$ \\
Sense 5'- TGTCGCTGTCTTGCACTCTG -3' & $p 21$ \\
Antisense 5'-CCAATCTGCGCTTGGAGTGATA -3' & $p 21$ \\
Sense 5'- TGCTCACCCTGGCTAAAGTT -3' & $p 53$ \\
Antisense 5'-GTCCATGCAGTGAGGTGATG -3' & $p 53$ \\
Sense 5'- ATGAACTTTCTGCTCTCTTGGGT -3' & $V E G F-A$ \\
Antisense 5'-CACAGGACGGCTTGAAGATGTA -3' & $V E G F-A$ \\
Sense 5'- TGCTGGTCATGAAGCTGTTC -3' & $P l G F$ \\
Antisense 5'-GGACACAGGACGGACTGAAT -3' & $P l G F$ \\
Sense 5'- GACGATGAACATCAAGTCAGCA -3' & $H I F l \alpha$ \\
Antisense 5'-GGAATGGGTTCACAAATCAGCAC -3' & $H I F l \alpha$ \\
Sense 5'- GAGGAGGATGAGGGTGTCTATAG -3' & $F l t-1$ \\
Antisense 5'-TGATCAGCTCCAGGTTTGACT -3' & $F l t-1$ \\
Sense 5'- CTTCCAAGGACAGCCAAGAGT -3' & $E N G$ \\
Antisense 5'-GTGGTTGCCATTCAAGTGTGG -3' & $E N G$ \\
Sense 5'- TCGAAGACATGCCGTTGGG -3' & $I N H A$ \\
Antisense 5'-AGCTGGCTGGTCCTCACA -3' & $I N H A$ \\
\hline
\end{tabular}




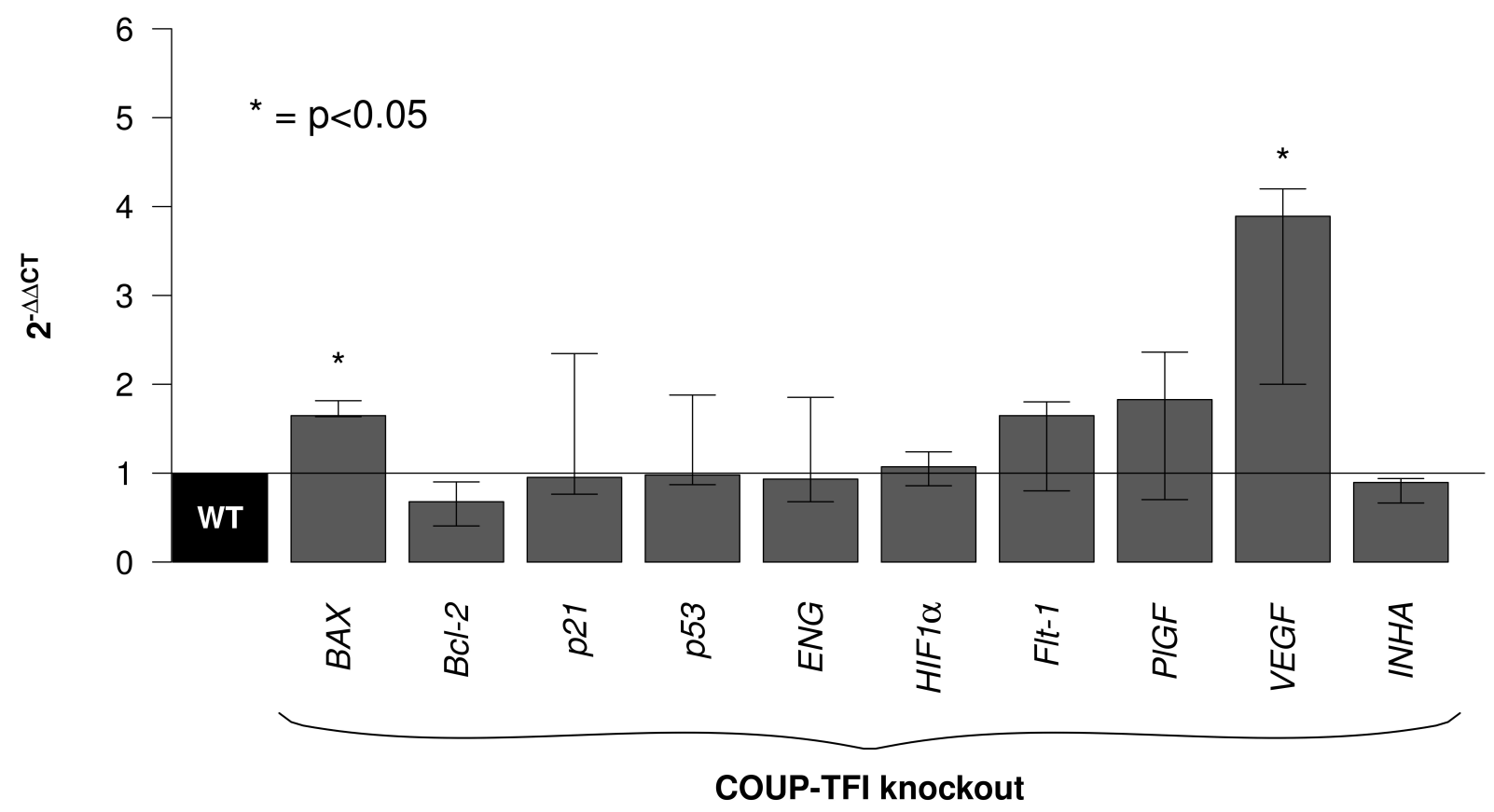

Fig. 1. Key placental genes expression pattern. Box plot showing fold change values $\left(2^{-\Delta \Delta C t}\right)$ of key placental genes in COUP-TFI $K O$ (grey boxes) compared to $W T$ (black box) levels. The values refer to the median and IQR and the p-values to the Wilcoxon test.

The performed reactions were run in triplicate in three independent experiments. The $m R N A$ quantification was expressed in terms of the cycle threshold $(\mathrm{Ct})$. From each triplicate run, the means of the $\mathrm{Ct}$ values were calculated and used for further analysis. All gene expression levels were normalized on the values of the housekeeping gene glyceraldehyde-3phosphate dehydrogenase $(G A P D H)$. Differences between the $\mathrm{Ct}$ values of the tested genes and those of the reference gene were calculated as $\Delta \mathrm{Ct}($ gene $)=\mathrm{Ct}($ gene $)-$ $\mathrm{Ct}(G A P D H)$ and represented as $2^{(-\Delta C t)}$ values. The relative fold changes in expression levels were determined as $2^{(-\Delta \Delta C t)}$ that was determined by the following equation: $2^{(-(\Delta C t(\text { gene in } K O)-\Delta C t(\text { gene in } W T)))}$.

\subsection{Statistical analysis}

Data were analyzed using $\mathrm{R}$ v3.5.3 with $p<0.05$ considered as significant. Based on preliminary data, a sample size of 4 mice per group, with $\alpha$ of 0.05 and $80 \%$ power, was found adequate to detect as significant a 1.5 fold difference in the expression of the targeted genes using a nonparametric test. To compensate for possible missing data, a total of 5 mice per group was considered. Distribution normality was tested by the Kolmogorov-Smirnov test. The $T$-test, Mann-Whitney-test, and Spearman test were performed as appropriate.

\section{Results}

According to RT-qPCR data, the most highly expressed gene in both COUP-TFI KO and WT placental tis- sue was $H I F 1 \alpha$, followed by Bax and $p 21$. In addition, $p 21$ resulted more expressed than $p 53$, in both COUP-TFI KO and $W T$ placental tissues (not shown). Interestingly, we found an increase of $V E G F-A$ and $B a x$ mRNA expression in placental tissue of COUP-TFI KO mice compared to their control counterparts. On the contrary, no significant differences were observed in mRNA expression of other marker of placental development, such as Flt-1, PlGF, Bcl-2, p21, p53, VEGF-A, HIF $1 \alpha, E N G$, and INHA (Fig. 1 and Table 2).

Table 2. Expression delta-CT values $\left(2^{(-\Delta C T)}\right)$ of the studied mRNAs. The values are reported as median and IRQ and the $p$-values refer to the Wilcoxon test.

\begin{tabular}{lccc}
\hline & COUP-TFI KO & Wilde type $(W T)$ & $p$ \\
\hline BAX & $0.035(0.033-0.036)$ & $0.020(0.020-0.021)$ & $<0.05$ \\
Bcl-2 & $0.003(0.002-0.003)$ & $0.003(0.002-0.005)$ & 0.841 \\
$p 21$ & $0.031(0.023-0.047)$ & $0.020(0.017-0.051)$ & 1.000 \\
$p 53$ & $0.001(0.000-0.001)$ & $0.000(0.000-0.001)$ & 0.690 \\
ENG & $0.004(0.004-0.010)$ & $0.008(0.003-0.011)$ & 1.000 \\
HIF1 $\alpha$ & $0.047(0.045-0.071)$ & $0.061(0.037-0.073)$ & 1.000 \\
Flt1 & $0.009(0.007-0.018)$ & $0.010(0.005-0.010)$ & 1.000 \\
PlGF & $0.015(0.011-0.020)$ & $0.008(0.004-0.030)$ & 0.548 \\
VEGF1 & $0.010(0.006-0.011)$ & $0.003(0.002-0.005)$ & $<0.05$ \\
INHA & $0.003(0.001-0.003)$ & $0.001(0.001-0.003)$ & 1.000 \\
\hline
\end{tabular}

Correlations between all evaluated transcripts in $W T$ and COUP-TFI KO mice are shown in Fig. 2. We found significant positive correlations in the placental tissue of 

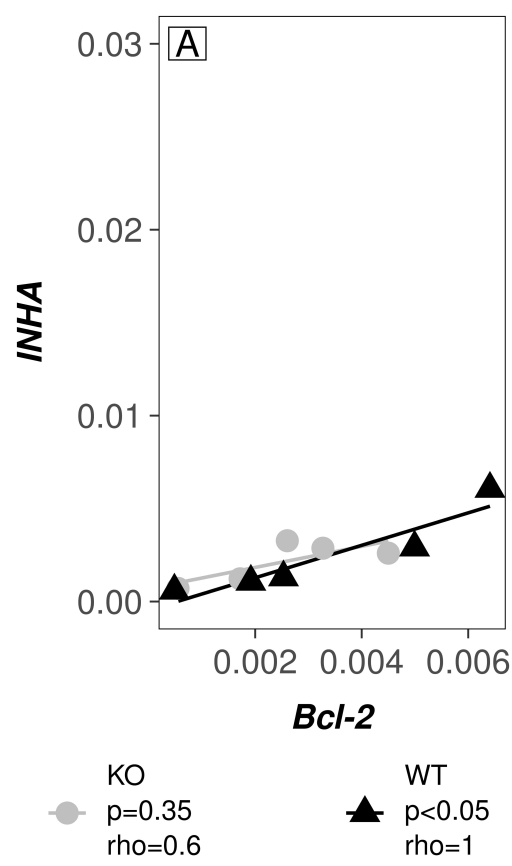
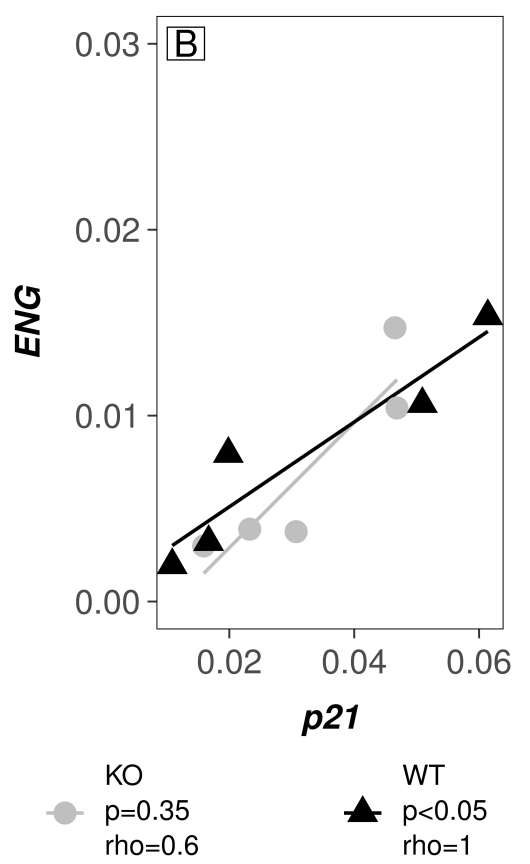

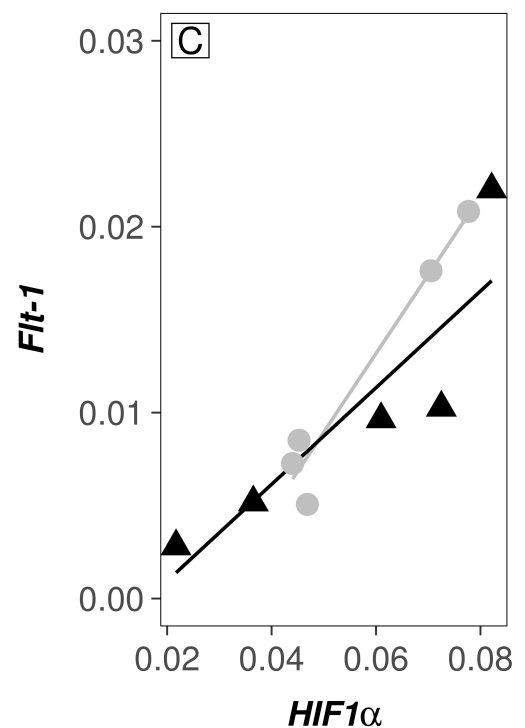

$\mathrm{KO}$

$p=0.35$

rho $=0.6$
WT

$\wedge \mathrm{p}<0.05$

rho $=1$

Fig. 2. Plots showing correlations between placental genes in $\boldsymbol{W T}$ and $\boldsymbol{C O U P}$-TFI KO mice. Panel (A) Shows correlation between INHA and Bcl-2. Panel (B) Shows correlation between ENG and p21. And Panel (C) Shows correlation between HIFl $\alpha$ and Flt-1. $p$-values and rho refer to Spearman test.

WT mice between the following mRNA pairs: $B c l-2$ and INHA (rho $=1$ and $p<0.05$ ); $p 21$ and $E N G$ (rho $=1$ and $p<0.05$ ); HIFl $\alpha$ and Flt-1 (rho $=1$ and $p<0.05$ ). Notably, such positive correlation between $B c l-2$ and INHA, p21 and ENG and between HIFI $\alpha$ and Flt-1 mRNA expression was lost in mutant placentas. Additional positive correlations were found in WT placental tissue between the following pairs, even though they did not reach statistical significance (not shown): $p 21$ and $p 53$ (rho $=0.9$ and $p=$ 0.083 ); $p 21$ and HIF1 $\alpha$ (rho $=0.9$ and $p=0.083$ ); $p 21$ and Flt-1 (rho $=0.9$ and $p=0.083$ ); $p 21$ and PlGF (rho $=0.9$ and $p=0.083$ ); $E N G$ and $p 53$ (rho $=0.9$ and $p=0.083$ ); $E N G$ and HIFl $\alpha$ (rho $=0.9$ and $p=0.083) ; E N G$ and Flt-1 (rho $=0.9$ and $p=0.083$ ); and $E N G$ and $P l G F$ (rho $=0.9$ and $p=0.083$ ). All tested correlations were no longer significant in the placental tissue of COUP-TFI KO samples, where we only found a negative correlation between $\mathrm{Bcl}-2$ and $P l G F$, even though not significant (rho $=-0.9$ and $p=$ 0.083 ).

Finally, we found a reduction of weight in COUPTFI KO pups compared to littermates controls. The mean weight of WT mice was 1.6 grams $( \pm 0.14)$, compared to 1.3 grams $( \pm 0.13)$ of COUP-TFI KO mice $(p<0.05)$.

\section{Discussion}

In this study, we assessed the role of mouse COUP$T F I$ in regulating the expression of major genes involved in placentation. We found that $V E G F-A$ and Bax mRNA expression were increased in COUP-TFI KO compared to wild-type mouse placentas, suggesting an impairment of apoptotic and angiogenetic pathways in mutant placental tissue. The positive correlations observed in normal placental tissue between $B c l-2$ and $I N H A, p 21$ and $E N G$, and HIF $1 \alpha$ and Flt- 1 were lost in COUP-TFI mutants, suggesting that key molecular networks could be imbalanced upon COUP-TFI loss. Interestingly, mutant mice also showed a significantly lower weight at birth compared to wildtype mice, raising the possibility that placental impairments described above could ultimately converge in sub-optimal weight gain during gestation.

We focused our attention on COUP-TFI because this family of nuclear receptors carries out vital roles in physiological processes, including proliferation, apoptosis and cell signaling $[15,20]$. Thanks to the level of evolutionary conservation of COUP-TFI, understanding the pathological pathway in relation to its expression in mouse models could help to better focus future studies on genes known to be relevant during the process of physiological placental development. In particular, we analyzed the expression of $H I F 1 \alpha, E N G, F l t 1, P l G F, V E G F-A$, which are main players in angiogenesis and vascular pathfinding, $p 21$ and $p 53$ in cellular proliferation, as well as Bax, Bcl2 and INHA, which are involved in apoptosis and cell survival. All these genes regulate crucial aspects of placental development, and their variation was shown to be associated with impaired development of the ongoing pregnancy [21,22].

When considering the influence of COUP-TFI on the expression of the most common angiogenetic factors, we found that $V E G F-A$ mRNA was consistently up-regulated 
in COUP-TFI KO placentas compared to control mice. The isoform A of the vascular endothelial growth factor ( $V E G F$ $A$ ), belonging to the VEGF family, is considered the most crucial factor promoting the differentiation of mesenchymal cells in villi into hemangioblastic stem cells. $V E G F-A$ expression is induced by hypoxia, as a potent stimulus, and is mediated via HIF $1 \alpha$ expression [23-26]. Indeed, $V E G F-A$ is strongly expressed by the cytotrophoblast cells during the first trimester of pregnancy and strong evidence indicates that high $V E G F-A$ expression in fetal growth restriction reflects the hypoxic status of the placenta [27,28].

Supporting this evidence, we found in COUP-TFI mutants a loss in the positive correlation between $m R N A$ expression of HIFl $\alpha$ and Flt-1, this latter encoding the vascular endothelial growth factor receptor 1 (VEGFR1), one of the receptors for vascular endothelial growth factors $(V E G F)$. In a hypoxic environment, Hifl $\alpha$ could regulate the expression of $V E G F-A, F l t-1$ and other angiogenic factors, by means of a compensatory mechanism aimed to restore normal placental blood flow and to rescue normoxia [29,30]. This finding overlaps with studies showing that VEGF-A $m R N A$ and protein levels are significantly reduced in patients with growth restriction and that an adenovirus-mediated overexpression of VEGF can improve fetal growth in a sheep model $[31,32]$. Regarding the other angiogenic factors considered in our study, $P l G F$ and $E N G$, no significant differences were found in COUP-TFI $K O$ placentas compared to control mice.

Considering the most common genes involved in cell proliferation and survival control, we observed an increase of Bax mRNA in mutant placentas. An augmented Bax expression is in line with other studies conducted on human placenta [33-35]. Bax is a pro-apoptotic protein that exerts, in concert with the anti-apoptotic protein $\mathrm{Bcl}-2$, a crucial role in apoptosis. Both are regulated by the $p 53$ tumor suppressor gene [36]. Apoptosis contributes to the turnover of villous trophoblasts and plays a crucial function in the remodeling of spiral arteries in human placenta. Apoptosis in placental villi changes throughout normal pregnancy: it is low in the first trimester, increases in the second, and then reaches the highest levels beyond 40 weeks of gestation [37]. Furthermore, the amount of apoptosis is increased in villous trophoblast in placental pathologies, including preeclampsia [38].

In addition, we observed a significant positive correlation of $B c l-2$ and INHA in normal mice. This could be due to a regulatory role of on trophoblast growth through inhibition of the activin receptor, known to have a fundamental role in trophoblast development and correct placentation [39]. This, in turn, could result in reduced placenta proliferation and increased apoptosis characterizing old placentas at the end of gestation [40]. According to our study, this correlation seems to be altered by the absence of COUPTFI.

Interestingly, we also observed a lower level of $p 53$ expression than its downstream target $p 21$ in COUP-TFI $K O$ samples, and a positive correlation of the expression rate of the two genes. These data confirmed previous results on human placentas [21]. We can further hypothesize a role of $p 21$ independent of $p 53$. Usually $p 53$, through $p 21$, promotes cell cycle arrest or apoptosis via the augmented expression of $\operatorname{Bax}[36,41]$. In the current study, we found a significant positive correlation between $p 21$ and ENG transcripts. $E N G$ is part of the transforming growth factor-beta receptor complex. Angiogenesis, apoptosis, and cell cycle arrest could be promoted by the transforming growth factorbeta pathway, reported to be implicated in fetal growth restriction [42]. p21 could also possibly interact in placental tissue with this cascade triggered by the transforming growth factor-beta receptor complex [43]. As the correlation between $p 53$ and $p 21$ is lost in mutant mice, this interaction could be disturbed by the absence of COUP-TFI.

Finally, we looked at the link of the mouse phenotype to placental function in terms of weight of pups recorded at birth. Interestingly, our results showed that COUP-TFI KO pups presented a significant lower weight than WT littermate controls. These data further support the pathological significance of COUP-TFI in placental development, potentially related to fetal growth restriction, a common complication associated with impaired placental function in humans $[9,44,45]$. Our preliminary results invite further studies on specific downstream cascades of molecular markers linked to COUP-TFI, both in mouse models and humans.

This explorative study aimed to identify potential markers involved in impaired placental function linked to COUP-TFI loss-of-function but lacks a detailed analysis of mechanisms underlying the downstream regulation of angiogenic, cell regulation and apoptotic factors included in this mouse model. Moreover, in our experiments we have not assessed the function COUP transcription factor 2 (COUP-TFII), a homolog to COUP-TFI sometimes compensating COUP-TFI functions. COUP-TFI and COUP$T F I I$ expression patterns overlaps in many regions and organs, possibly resulting in redundant functions $[6,46,47]$. Thus, COUP-TFII may be able to compensate for the absence of COUP-TFI in COUP-TFI KO mice. Further experiments on placentas lacking both COUP-TF members derive from crossing between COUP-TFI and COUP-TFII mutant mice, could shed new light on the interplay between these nuclear receptors during placental development. Exploring in more detail placental morphology could also be a topic of future interest.

\section{Conclusions}

The present study provides evidence that the absence of COUP-TFI influences the expression levels of two key effectors of mouse placental angiogenesis and apoptosis, $V E G F-A$ and Bax. Consistently, we showed that COUPTFI KO mice presented a significant lower weight at delivery than WT littermate controls. Hence, we propose 
that COUP-TFI plays an important role for placental development and function, even though further studies will be necessary to dissect the molecular dynamics governing a COUP-TFI-dependent placenta development.

\section{Abbreviations}

Bax, BCL2-associated X protein; Bcl-2, B-cell lymphoma 2; COUP-TFI, Chicken Ovalbumin Upstream Promoter-Transcription Factor I; COUP-TFII, Chicken Ovalbumin Upstream Promoter-Transcription Factor II; $E N G$, Endoglin; FLT-1, Fms-like tyrosine kinase 1 (also known as VEGFR1); HIF1 $\alpha$, Hypoxia inducible factor 1 alpha subunit; INHA, Inhibin alpha; mRNA, Messenger ribonucleic acid; $V E G F-A$ : Vascular Endothelial Growth Factor A.

\section{Author contributions}

LV, SM, APL, MS, LM, AF-substantial contributions to conception and design. LV, SM, MB, APL, MO, $\mathrm{SB}, \mathrm{MS}, \mathrm{LM}, \mathrm{AF}$ - substantial contributions to acquisition of data or to analysis and interpretation of data. LV, SM, MB, APL, MO, SB, LD, CDL, MS, LM, AF_-drafting the article or revising it critically for important intellectual content. All authors read and approved the final manuscript.

\section{Ethics approval and consent to participate}

All mouse experiments were conducted in accordance with the relevant national and international guidelines and regulations (European Union rules; 2010/63/UE), and with approval by the local ethical committee in France (CIEPAL NCE/2019-548).

\section{Acknowledgment}

The authors would like to thank Christian Alfano from iBV, Nice, France, for initial interest in this study and the Pathology of the AOU Santa Maria della Misericordia, University of Udine, Italy, who collaborated in the study.

\section{Funding}

This research received no external funding.

\section{Conflict of interest}

The authors declare no conflict of interest. APL is the Editor of this journal, given his role as Editor, had no involvement in the peer-review of this article and has no access to information regarding its peer-review.

\section{Availability of data and material}

The data that support the findings of this study are available, but restrictions apply to the availability of these data, which were used under license for the current study, and so are not publicly available. Data are however available from the authors upon reasonable request and with permission of the Internal Review Board.

\section{References}

[1] Burton GJ, Jauniaux E. What is the placenta? American Journal of Obstetrics and Gynecology. 2015; 213: S6.e1-S6.e4.

[2] Neerhof MG, Thaete LG. The fetal response to chronic placental insufficiency. Seminars in Perinatology. 2008; 32: 201-205.

[3] Park J, Tsai SY, Tsai M. Molecular mechanism of chicken ovalbumin upstream promoter-transcription factor (COUP-TF) actions. The Keio Journal of Medicine. 2003; 52: 174-181.

[4] Pereira FA, Tsai MJ, Tsai SY. COUP-TF orphan nuclear receptors in development and differentiation. Cellular and Molecular Life Sciences. 2000; 57: 1388-1398.

[5] Alfano C, Magrinelli E, Harb K, Studer M. The nuclear receptors COUP-TF: a long-lasting experience in forebrain assembly. Cellular and Molecular Life Sciences. 2014; 71: 43-62.

[6] Qiu Y, Cooney AJ, Kuratani S, DeMayo FJ, Tsai SY, Tsai MJ. Spatiotemporal expression patterns of chicken ovalbumin upstream promoter-transcription factors in the developing mouse central nervous system: evidence for a role in segmental patterning of the diencephalon. Proceedings of the National Academy of Sciences of the United States of America. 1994; 91: 44514455.

[7] Tsai SY, Tsai MJ. Chick ovalbumin upstream promotertranscription factors (COUP-TFs): coming of age. Endocrine Reviews. 1997; 18: 229-240.

[8] Genbacev O, Donne M, Kapidzic M, Gormley M, Lamb J, Gilmore J, et al. Establishment of human trophoblast progenitor cell lines from the chorion. Stem Cells. 2012; 29: 1427-1436.

[9] Vaiman D, Calicchio R, Miralles F. Landscape of transcriptional deregulations in the preeclamptic placenta. PLoS ONE. 2013; 8: e65498.

[10] Abrahamsohn PA, Zorn TM. Implantation and decidualization in rodents.The Journal of Experimental Zoology. 1993; 266: 603628.

[11] Georgiades P, Ferguson-Smith AC, Burton GJ. Comparative developmental anatomy of the murine and human definitive placentae. Placenta. 2002; 23: 3-19.

[12] Simmons DG, Cross JC. Determinants of trophoblast lineage and cell subtype specification in the mouse placenta. Developmental Biology. 2005; 284: 12-24.

[13] Ko MS. Embryogenomics: developmental biology meets genomics. Trends in Biotechnology. 2001; 19: 511-518.

[14] Armentano M, Filosa A, Andolfi G, Studer M. COUP-TFI is required for the formation of commissural projections in the forebrain by regulating axonal growth. Development. 2006; 133: 4151-4162.

[15] Bertacchi M, Parisot J, Studer M. The pleiotropic transcriptional regulator COUP-TFI plays multiple roles in neural development and disease. Brain Research. 2019; 1705: 75-94.

[16] Armentano M, Chou S, Tomassy GS, Leingärtner A, O'Leary DDM, Studer M. COUP-TFI regulates the balance of cortical patterning between frontal/motor and sensory areas. Nature Neuroscience. 2007; 10: 1277-1286.

[17] Harb K, Magrinelli E, Nicolas CS, Lukianets N, Frangeul L, Pietri M, et al. Area-specific development of distinct projection neuron subclasses is regulated by postnatal epigenetic modifications. ELife. 2016; 5: e09531.

[18] Kilkenny C, Browne WJ, Cuthill IC, Emerson M, Altman DG Improving bioscience research reporting: the ARRIVE guidelines for reporting animal research. PLoS Biology. 2010; 8: e1000412.

[19] Natale DRC, Starovic M, Cross JC. Phenotypic analysis of the mouse placenta. Methods in Molecular Medicine. 2006; 121: 275-293.

[20] Schoorlemmer J, Jonk L, Sanbing S, van Puijenbroek A, Feijen A, Kruijer W. Regulation of Oct-4 gene expression during dif- 
ferentiation of EC cells. Molecular Biology Reports. 1995; 21: 129-140.

[21] Londero AP, Orsaria M, Marzinotto S, Grassi T, Fruscalzo A, Calcagno A, et al. Placental aging and oxidation damage in a tissue micro-array model: an immunohistochemistry study. Histochemistry and Cell Biology. 2016; 146: 191-204.

[22] Louwen F, Muschol-Steinmetz C, Reinhard J, Reitter A, Yuan J. A lesson for cancer research: placental microarray gene analysis in preeclampsia. Oncotarget. 2012; 3: 759-773.

[23] Carmeliet P, Ferreira V, Breier G, Pollefeyt S, Kieckens L, Gertsenstein $\mathrm{M}$, et al. Abnormal blood vessel development and lethality in embryos lacking a single VEGF allele. Nature. 1996; 380: 435-439.

[24] Ferrara N, Carver-Moore K, Chen H, Dowd M, Lu L, O'Shea $\mathrm{KS}$, et al. Heterozygous embryonic lethality induced by targeted inactivation of the VEGF gene. Nature. 1996; 380: 439-442.

[25] Fukushima K, Murata M, Hachisuga M, Tsukimori K, Seki H, Takeda S, et al. Hypoxia inducible factor 1 alpha regulates matrigel-induced endovascular differentiation under normoxia in a human extravillous trophoblast cell line. Placenta. 2008; 29: 324-331.

[26] Taylor CM, Stevens H, Anthony FW, Wheeler T. Influence of hypoxia on vascular endothelial growth factor and chorionic gonadotrophin production in the trophoblast-derived cell lines: JEG, JAr and BeWo. Placenta. 1997; 18: 451-458.

[27] Sgambati E, Marini M, Zappoli Thyrion GD, Parretti E, Mello $\mathrm{G}$, Orlando $\mathrm{C}$, et al. VEGF expression in the placenta from pregnancies complicated by hypertensive disorders. BJOG: an International Journal of Obstetrics and Gynaecology. 2004; 111: 564-570.

[28] Tsatsaris V, Goffin F, Munaut C, Brichant J, Pignon M, Noel $\mathrm{A}$, et al. Overexpression of the soluble vascular endothelial growth factor receptor in preeclamptic patients: pathophysiological consequences. The Journal of Clinical Endocrinology and Metabolism. 2003; 88: 5555-5563.

[29] Forsythe JA, Jiang BH, Iyer NV, Agani F, Leung SW, Koos RD, et al. Activation of vascular endothelial growth factor gene transcription by hypoxia-inducible factor 1. Molecular and Cellular Biology. 1996; 16: 4604-4613.

[30] Manalo DJ, Rowan A, Lavoie T, Natarajan L, Kelly BD, Ye SQ, et al. Transcriptional regulation of vascular endothelial cell responses to hypoxia by HIF-1. Blood. 2005; 105: 659-669.

[31] Alahakoon TI, Zhang W, Arbuckle S, Zhang K, Lee V. Reduced angiogenic factor expression in intrauterine fetal growth restriction using semiquantitative immunohistochemistry and digital image analysis. The Journal of Obstetrics and Gynaecology Research. 2018; 44: 861-872.

[32] Carr DJ, Wallace JM, Aitken RP, Milne JS, Mehta V, Martin JF, et al. Uteroplacental Adenovirus Vascular Endothelial Growth Factor Gene Therapy Increases Fetal Growth Velocity in Growth-Restricted Sheep Pregnancies. Human Gene Therapy. 2014; 25: 375-384

[33] Börzsönyi B, Demendi C, Rigó J, Szentpéteri I, Rab A, Joó JG. The regulation of apoptosis in intrauterine growth restriction: a study of Bcl-2 and Bax gene expression in human placenta. The Journal of Maternal-Fetal \& Neonatal Medicine. 2013; 26: 347 350.

[34] Cobellis L, De Falco M, Torella M, Trabucco E, Caprio F, Fed- erico E, et al. Modulation of Bax expression in physiological and pathological human placentas throughout pregnancy. In Vivo. 2007; 21: 777-783.

[35] Levy R, Smith SD, Yusuf K, Huettner PC, Kraus FT, Sadovsky $\mathrm{Y}$, et al. Trophoblast apoptosis from pregnancies complicated by fetal growth restriction is associated with enhanced p53 expression. American Journal of Obstetrics and Gynecology. 2002; 186: 1056-1061.

[36] Miyashita T, Reed JC. Tumor suppressor p53 is a direct transcriptional activator of the human bax gene. Cell. 1995; 80: 293299.

[37] Athapathu H, Jayawardana MAJ, Senanayaka L. A study of the incidence of apoptosis in the human placental cells in the last weeks of pregnancy. Journal of Obstetrics and Gynaecology. 2003; 23: 515-517.

[38] Leung DN, Smith SC, To KF, Sahota DS, Baker PN. Increased placental apoptosis in pregnancies complicated by preeclampsia. American Journal of Obstetrics and Gynecology. 2001; 184: $1249-1250$.

[39] Muttukrishna S, Bearfield C, Johns J, Jauniaux E. Inhibin, activin, follistatin, activin receptors and beta-glycan gene expression in the villous tissue of miscarriage patients. Molecular Human Reproduction. 2004; 10: 793-798.

[40] Walton KL, Makanji Y, Wilce MC, Chan KL, Robertson DM, Harrison CA. A common biosynthetic pathway governs the dimerization and secretion of inhibin and related transforming growth factor beta (TGFbeta) ligands. The Journal of Biological Chemistry. 2009; 284: 9311-9320.

[41] Sharp AN, Heazell AEP, Baczyk D, Dunk CE, Lacey HA, Jones CJP, et al. Preeclampsia is associated with alterations in the $\mathrm{p} 53$ pathway in villous trophoblast. PLoS ONE. 2014; 9: e87621.

[42] Lyall F, Simpson H, Bulmer JN, Barber A, Robson SC. Transforming growth factor-beta expression in human placenta and placental bed in third trimester normal pregnancy, preeclampsia, and fetal growth restriction. The American Journal of Pathology. 2001; 159: 1827-1838.

[43] Yoo YD, Choi JY, Lee SJ, Kim JS, Min BR, Lee YI, et al. TGFbeta-induced cell-cycle arrest through the p21(WAF1/CIP1)-G1 cyclin/Cdks-p130 pathway in gastric-carcinoma cells. International Journal of Cancer. 1999; 83: 512-517.

[44] Fruscalzo A, Londero AP, Orsaria M, Marzinotto S, Driul L, Di Loreto $\mathrm{C}$, et al. Placental fibronectin staining is unaffected in pregnancies complicated by late-onset intrauterine growth restriction and small for gestational age fetuses. International Journal of Gynecology \& Obstetrics. 2020; 148: 253-260.

[45] Londero AP, Bertozzi S, Visentin S, Fruscalzo A, Driul L, Marchesoni D. High placental index and poor pregnancy outcomes: a retrospective study of 18,386 pregnancies. Gynecological Endocrinology. 2013; 29: 666-669.

[46] Jonk LJ, de Jonge ME, Pals CE, Wissink S, Vervaart JM, Schoorlemmer J, et al. Cloning and expression during development of three murine members of the COUP family of nuclear orphan receptors. Mechanisms of Development. 1994; 47: 81-97.

[47] Pereira FA, Qiu Y, Tsai M, Tsai SY. Chicken ovalbumin upstream promoter transcription factor (COUP-TF): Expression during mouse embryogenesis. The Journal of Steroid Biochemistry and Molecular Biology. 1995; 53: 503-508. 